\title{
Editorial
}

\section{Frontiers in the Convergence of Bioscience and Information Technology}

\author{
Daniel Howard \\ QinetiQ Limited, Malvern Technology Centre, St Andrew's Road, Malvern, Worcestershire WR14 3PS, UK
}

Correspondence should be addressed to Daniel Howard, dr.daniel.howard@gmail.com

Received 15 June 2008; Accepted 15 June 2008

Copyright (c) 2008 Daniel Howard. This is an open access article distributed under the Creative Commons Attribution License, which permits unrestricted use, distribution, and reproduction in any medium, provided the original work is properly cited.

This special issue places its emphasis on the crossroads between computational modeling and the biosciences. It is ambitious but fruitful interdisciplinary research, and this special issue aims to illustrate different presentations of it. It offers those advancing knowledge in some particular area the view to related research activity and opportunities.

Many of the articles exemplify how mathematics and computer simulation inform experimentation to obtain new knowledge about nature. Some articles represent discoveries and others review, introduce, test, or trial algorithms or tools that are potentially helpful to arrive at discoveries. A few articles, however, are reversed in that they demonstrate how bioinspired algorithms or biological devices can solve hard computational challenges. Consider that manipulating chemicals may compute an answer much faster than by means of the standard silicon-based computer and within this special issue there is an article by $\mathrm{K}$. Li et al. describing the principles by which such a DNA computer can compromise an encryption algorithm that is central to present day secure communications.

One might classify the articles in this special issue into three groupings: (1) those concerned with biological problems from molecular cell biology to systems biology, (2) those which apply classification algorithms in cancer diagnosis and methods of computer vision to anatomy, and finally (3) those which offer new knowledge or possibilities in bioengineering and biomedicine.

The first grouping covers Systems Biology, an exciting interdisciplinary field that marries experiments with computer simulations. Synthetic and systems biotechnology, for example, is a technology at these frontiers that aims to sequester the services of micro-organisms for the benefit of mankind. Its ability to produce foods in a vat that would otherwise take up valuable land resources with conventional agriculture will offer flexibility in food production. In their article, M.-J. Han et al. reveal how knowledge and understanding of cell physiology in the presence of oleic acid are obtained for E. coli. Interesting research about signaling networks in retina, as induced by light exposure in mice, is presented by J. Krishnan et al. showing marked alterations in gene expression upon light exposure. Certain transcription factors are discovered to be important for the responses to light-induced retinal loss, revealing that many of the apoptosis-related genes are up- or downregulated in this process. In their article, R. Moreno-Sanchez et al. offer a very useful review of metabolic control analysis (MCA), a tool that represents a type of engineering control theory for cell biology and when applicable MCA can help to grapple with an understanding of the complex control of the metabolic pathways.

Also in the first grouping, three articles by S. Huang and his colleagues develop the emergent and interdisciplinary field of infectomics, which is the study of infectomes encoded by the genomes of microbes and their hosts. Infectomics has potential to advance the rational strategies that will prevent and treat infectious diseases as these could require a full appreciation of the infectomes that contribute to microbial infections. There is a need to figure out how to dissect the dynamic duality relationship between symbiosis and pathogenesis in microbial infections, and advocates of this new field oppose what they see as the misguided, though current and popular, reductionist and Manichean views of the microbe-human host relationship. Another article on the topic of infectious diseases by K.-Y. Hwa et al. investigates the emerging and life-threatening infectious disease known as SARS, where there is a compelling need 
for the development of effective therapeutics. Their article presents interesting and potentially important findings, that molecular mimicry occurs between SARS-CoV and host proteins. They investigate how to predict those peptides that are worthy of exploration for their biological activity. The article by L. Hamel et al. provides novel insight into the field of phylogenetics with the idea of the spectra of a tree, and reviewers identified its potential to compare with phylogenetic trees across different genes and for detecting lateral gene transfer.

The first grouping of articles also covers specialist numerical algorithms for gene sequencing, genomics, and proteomics. With genomic sequencing, an error in contig assembly causes serious error proliferation, and ConPath (P.G. Kim et al.) is a tool that can address this problem. It constructs scaffolds, ordering and orienting separate sequence contigs by exploiting the mate-pair information between contig pairs. C.-K. Chan et al. develop the growing selforganized map (GSOM) for binning (the clustering of these unassembled DNA sequences). They report improvements over the binning that combines oligonucleotide frequency and self-organizing maps (SOMs), and identify suitable training features and quantitative measures for assessing results in this area. In their article, $\mathrm{K}$. Han et al. develop an algorithm to search for the highly connected subgraphs in protein interaction networks because this might be helpful to predict protein function. The article by Mi-Young Kim expounds a new approach to the very important problem of text mining of biomolecular text, and is specifically concerned with detecting gene interactions. There follow two articles in biological chemistry with computational chemistry. C.-J. Kuo et al. solve the crystal structure of H. pylori undecaprenyl pyrophosphate synthase and perform virtual screening of inhibitors from a chemical library of thousands of compounds. M. Muddassar et al. explore receptor-guided 3D-QSAR to design IGF-1R inhibitors by pursuing careful statistical analysis to interpret the models that are obtained with the help of contour maps. The article by J.-C. Lue and W.-C. Fang proposes a compact integrated microsystem solution for robust, real-time, and onsite genetic analysis. It uses a preceding VLSI differential logarithm microchip that is designed to compute the logarithm of the normalized input fluorescence signals and a succeeding VLSI artificial neural network (ANN) processor chip to analyze the processed signals from the differential logarithm stage. It is submitted that the version of ANN chosen is particularly adept at recognizing the low-fluorescence patterns.

The second grouping includes cancer diagnosis, biomedical imaging and also computational anatomy (the study of anatomical variability in health and disease via deformable templates as inspired by D'Arcy Wentworth Thompson). These fields sit in the frontiers of biosciences, image analysis, mathematics, and numerical methods. In this grouping, a number of articles implement (D. Howard et al.), evaluate (N. A. Lee et al.), or introduce (J. Kolibal et al.) techniques of image analysis to cluster, segment, or analyze images, or extract information or enrich biomedical images. For example, J. Woo et al. discuss multimodal data integration for computer-aided ablation of atrial fibrillation. Y. Park et al. analyze the interpoint dissimilarity comparisons in the hippocampus shape space to distinguish between hippocampi of subjects with three conditions (clinically depressed, high risk, and control subject), and discover the high-risk population closer in shape space to the control population than to the clinically depressed population. Additionally, they find that the left hippocampi carry more information than do the right. In their article, N. A. Lee et al. examine performance in the segmentation of high-resolution MRI subvolumes containing hippocampus, prefrontal cortex, and occipital lobe, as acquired on different scanners. They offer evidence that the alternating kernel mixture algorithm outperforms alternatives on the ten datasets considered. The availability of powerful imaging and other sensors, the availability of information technology, and the nature of modern threats point to population biometrics as a topic of enormous current and future importance. Implementing biometrics well is hard and is not yet properly understood. The methodological paper by Y. N. Shin et al. proposes a formal performance evaluation model for a biometric recognition system. They also implement face recognition systems based on the proposed model. The model seems to be useful in terms of database availability, compliance with standards, and evaluation costs. The proposed formalism may gain traction for other biometrics, and it has the potential to inform strategies for population-based biomedical imaging performance evaluation and standardization. The last article of this second grouping by J. Wichard et al. evaluates how bioinspired and computational intelligence algorithms compare in providing reliable early cancer diagnosis.

The third grouping comprises articles which cover advances in biomechanics, biophysics, and biomedicine. It includes a detailed biomechanics study by J. S. Merritt et al. on the equine distal forelimb, which is a common location of injuries related to mechanical overload. These authors combine analysis with experiment in a fairly thorough but concise way to calculate the forces in the major tendons and joint reaction from kinematic and kinetic data of walking and trotting horses. Their findings point to the importance of muscle tendon wrapping when evaluating joint loading in the distal forelimb. The article by C. Handapangoda and M. Premaratne describes a novel numerical technique for modeling optical pulse propagation in inhomogeneous scattering and absorption cross-sections through weakly scattering biological tissues. The design of implantable electronic devices to interact with the nervous system is an active field, the development of an efficient system for longterm stimulation of the optic nerve is rather timely (e.g., it is required to evaluate the long-term safety of retinal implants), and the methodological report by J. A. Zhou et al. describes the design of a suprachoroidal electrical retinal stimulator for long-term application. Finally, two articles in the third category advance novel robots (H. Sawada et al.) and haptic solutions (K.-U. Kyung et al.) that interact with the human senses and which might prove helpful to the sensorially impaired.

Daniel Howard 

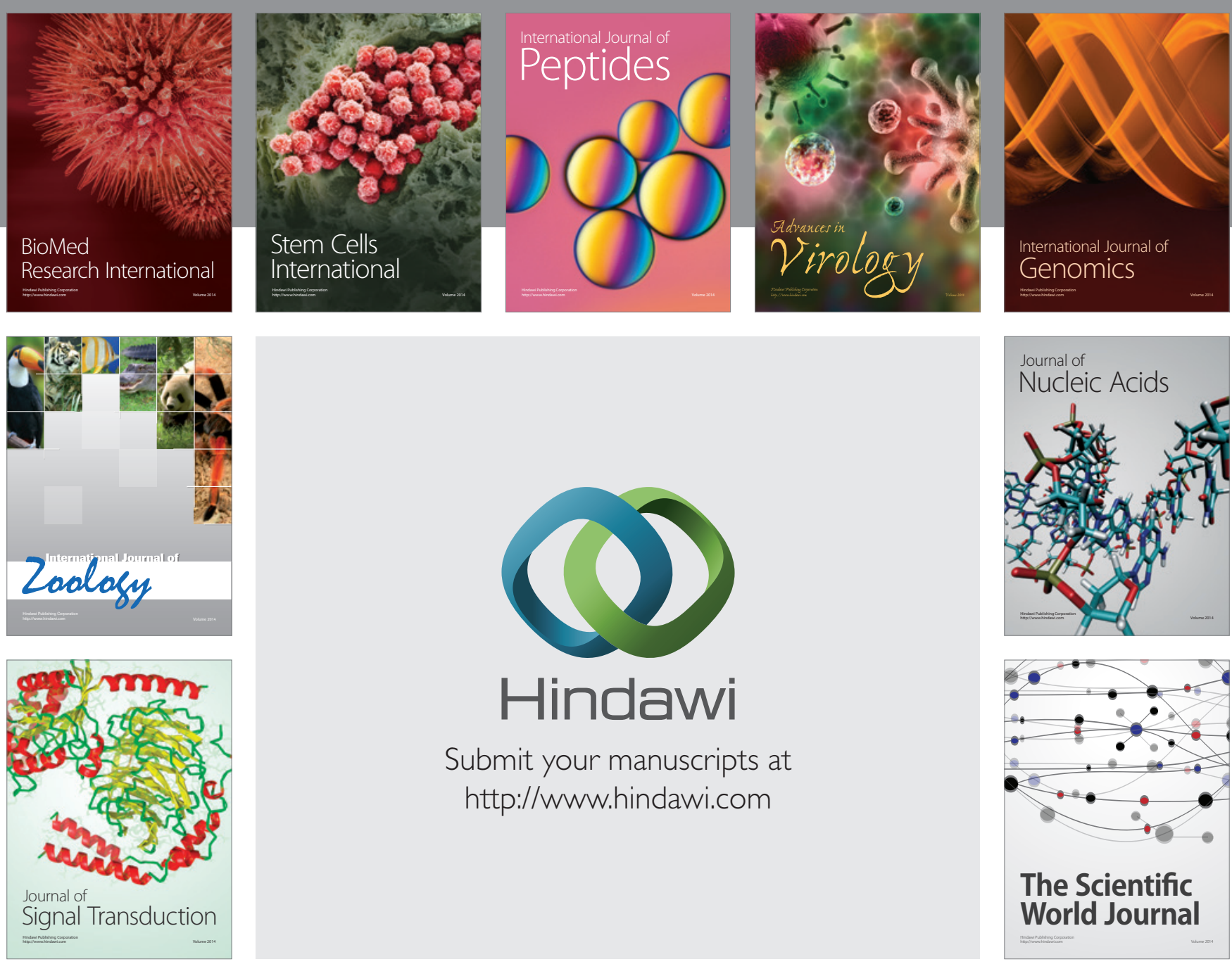

Submit your manuscripts at

http://www.hindawi.com
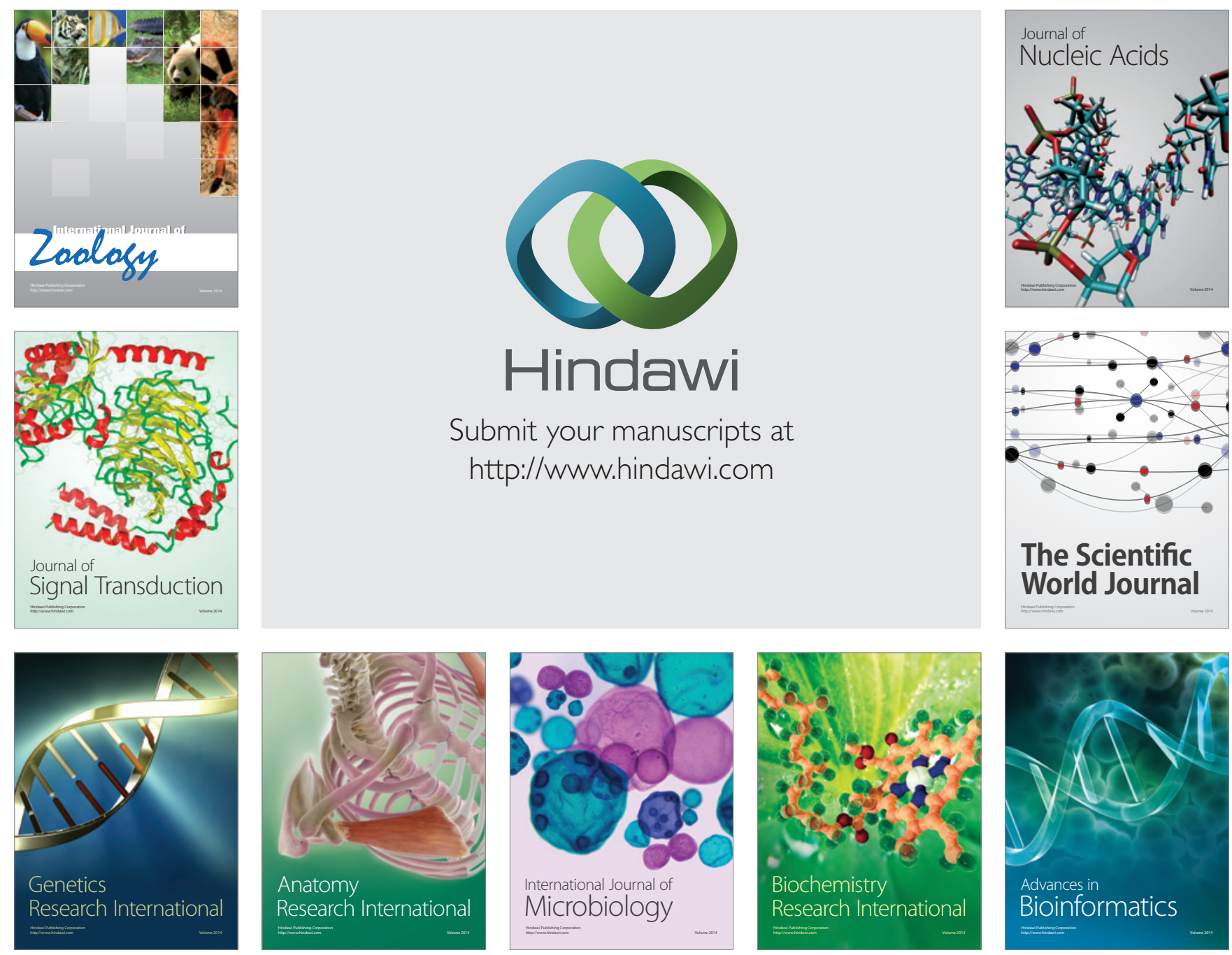

The Scientific World Journal
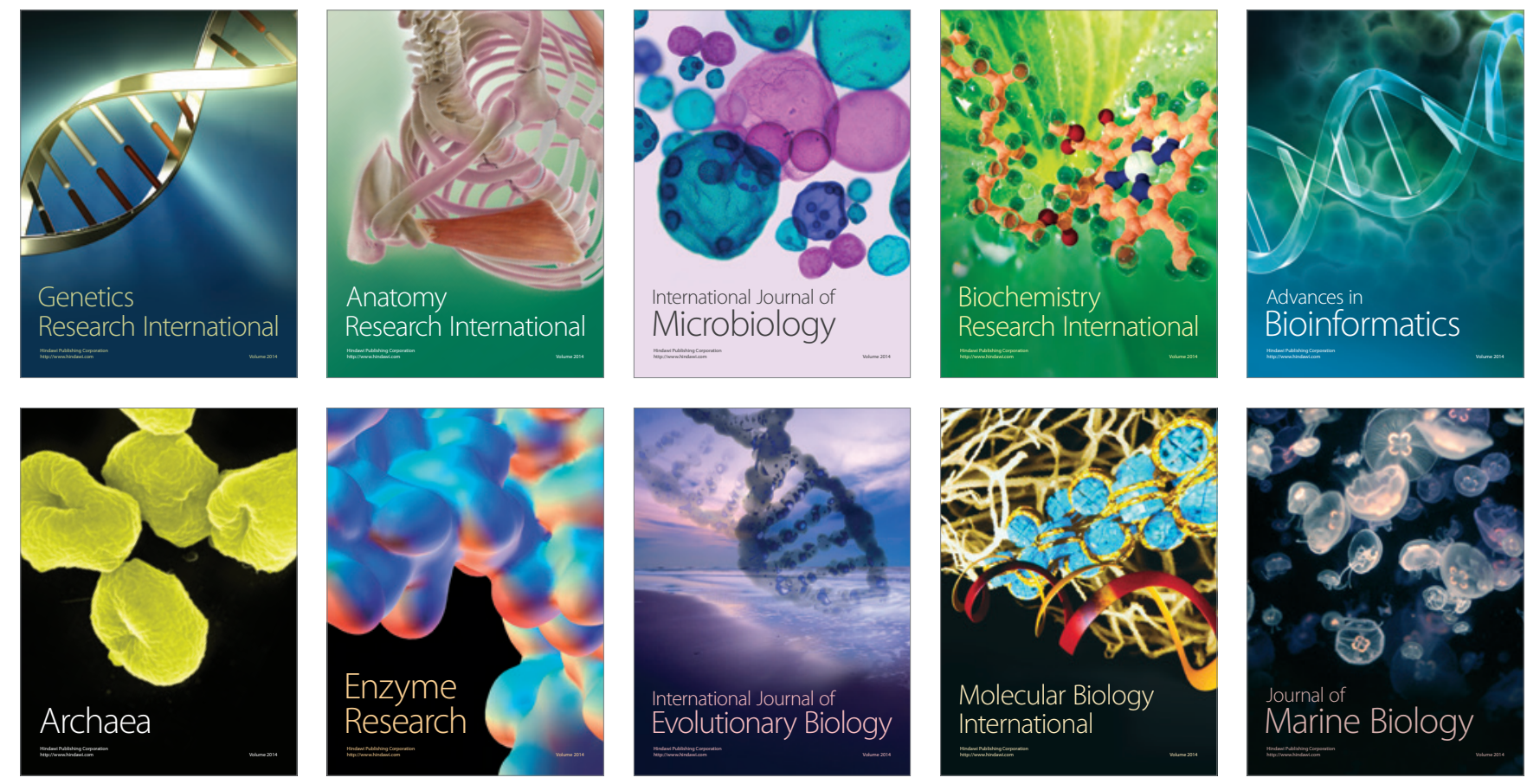